\title{
FEEDBACK ROBUST CONTROL FOR A PARABOLIC VARIATIONAL INEQUALITY
}

\author{
Vyacheslav Maksimov* \\ Institute of Mathematics and Mechanics \\ Ural Branch, Russian Academy of Sciences \\ Ekaterinburg, Russia \\ maksimov@imm.uran.ru
}

Abstract A problem of robust control of a parabolic variational inequality in the case of distributed control actions and disturbances is under consideration. The goal of the paper consists in the description and mathematical substantiation of the the method of feedback control in the formalization originated from works by N.N. Krasovskii [3], [2]. The paper continues investigations [5]-[4].

Keywords: robust control, parabolic variational inequality

\section{Introduction}

In the present work, the problem of robust control of distributed parameter systems is discussed. The essence of the problem under consideration may be formulated in the following way. A motion of a dynamical system $\Sigma$ proceeds on a given time interval $T=\left[t_{0}, \vartheta\right]$. System's trajectory $x(t)=x(t ; u(\cdot), v(\cdot)), t \in T$, depends on a time-varying unknown input $v=v(t) \in Q$, and a control $u=u(t) \in P$, where $P$ and $Q$ are given sets. Phase states of the system $\Sigma$ are inaccurately measured at time moments $\tau_{i} \in \Delta=\left\{\tau_{i}\right\}_{i=0}^{m}, \tau_{0}=t_{0}, \tau_{m}=\vartheta, \tau_{i+1}=\tau_{i}+\delta$. It is required to organize a process of control of the system $\Sigma$ by the feedback principle in such a way that it is possible to preserve given properties of system's trajectory under the action of any admissible input $v=v(\cdot)$.

\footnotetext{
* This work was supported in part by the Russian Foundation for Basic Research (grant \# 04-01-00059), Program on Basic Research of the Presidium of the Russian Acad. Sci. (project "Control of mechanical systems"), Program of supporting leading scientific schools of Russia (project 1846.2003.1) and Ural-Siberian Interdisciplinary Project.
} 
The quality of a trajectory constructed is estimated either by the distance from a given (prescribed, standard) trajectory $x_{*}(t)$ or by some quality functional (a payoff). The problem under discussion is treated as the problem of constructing a control $u=u(t)$ providing (under the action of any possible but unknown disturbance $v=v(t)$ ) retention of a trajectory $x(t)=x(t ; u(\cdot), v(\cdot))$ nearby $x_{*}(t)$ (in the first case) or minimizing maximally possible values of a payoff (in the second case). This is a meaningful formulation of the control problem under consideration.

\section{Statement of the Problem}

Let a system $\Sigma$ be described by the parabolic variational inequality

$$
\langle\dot{x}(t)+A x(t), x(t)-z\rangle+\varphi(x(t))-\varphi(z) \leq(f(t, u, v), x(t)-z)
$$

for a. a. $\quad t \in T$ and all $z \in V, \quad x\left(t_{0}\right)=x_{0}$.

Here $H=L_{2}(\Omega), V$ is a real Hilbert space, $V$ is a dense subspace of $H$ and $V \subset H \subset V^{*}$ algebraically and topologically, $(\cdot, \cdot)$ stands for the inner product in $H,\langle\cdot, \cdot\rangle$ stands for the duality relation between $V$ and $V^{*}, A: V \rightarrow V^{*}$ is a linear continuous $\left(A \in \mathcal{L}\left(V ; V^{*}\right)\right)$ and symmetrical operator satisfying (for some $\omega>0$ and real $\alpha_{0}$ ) the coercitivity condition

$$
(A x, x)+\alpha_{0}|x|_{H}^{2} \geq \omega|x|_{V}^{2} \quad \forall y \in V,
$$

$|\cdot|_{H}$ and $|\cdot|_{V}$ stand for the norm in $H$ and $V$, respectively, and $\varphi: V \rightarrow \bar{R}=\{r \in R:-\infty<r \leq+\infty\}$ is a lower semicontinuous convex function. Furthermore, without loss of generality, we assume that $\varphi(x) \geq 0 \quad \forall x \in V$. Let $x\left(t_{0}\right)=x_{0} \in D(\varphi)$, where $D(\varphi)=\{x \in V: \varphi(x)<+\infty\}$. Let $U_{p}$ and $U_{d}$ be uniformly convex Banach spaces, $f: T \times U_{p} \times U_{d} \rightarrow H$ be a Lipschitz function, $P$ and $Q$ be given bounded and closed sets from space of controls $U_{p}$ and disturbances $U_{d}$, respectively. It is known that under such conditions for any $\{u(\cdot), v(\cdot)\} \in L_{2}\left(T ; U_{p}\right) \times L_{2}\left(T ; U_{d}\right)$ there exists a unique solution $x(\cdot)=x\left(\cdot ; t_{0}, x_{0}, u(\cdot), v(\cdot)\right)$ of the inequality (1) with the following properties [1]: $x(\cdot)=x\left(\cdot ; t_{0}, x_{0}, u(\cdot), v(\cdot)\right) \in W_{*}(T)=W^{1,2}(T ; H) \cap L_{2}(T ; V)$, $x(t) \in D\left(\varphi_{\alpha}\right) \forall t \in T, t \rightarrow \varphi_{\alpha}(x(t)) \in A C(T)$. Here the function $\varphi_{\alpha}(y): H \rightarrow \bar{R}$ is defined by

$$
\varphi_{\alpha}(y)=\left\{\begin{array}{l}
1 / 2\langle A y, y\rangle+\alpha_{0} / 2|y|_{H}^{2}+\varphi(y), \text { if } y \in D(\varphi) \\
+\infty, \quad \text { otherwise, }
\end{array}\right.
$$

$W^{1,2}(T ; H)=\left\{w(\cdot) \in L_{2}(T ; H): w_{t}(\cdot) \in L_{2}(T ; H)\right\}$, the derivative $w_{t}(\cdot)$ is understood in the sense of distributions, $A C(T)$ is the set of absolutely continuous functions $x(t): T \rightarrow R$. 
Let us give some definitions. Furthermore, we denote by $u_{a, b}(\cdot)$ a function $u(t), t \in[a, b]$, considered as a whole. The symbol $P_{a, b}(\cdot)$ stands for restriction of a set $P_{T}(\cdot)$ onto the segment $[a, b] \subset T$. Any strongly measurable functions $u(\cdot): T \rightarrow P$ and $v(\cdot): T \rightarrow Q$ are called an open-loop control and a disturbance, respectively. The sets of all open-loop controls and disturbances are denoted by the symbols $P_{T}(\cdot)$ and $Q_{T}(\cdot)$. Elements of the product $T \times D(\varphi)$ are called positions. A unique solution of the inequality (1) with the properties $x\left(t_{*}\right)=$ $x_{*}, x(\cdot)=x\left(\cdot ; t_{*}, x_{*}, u_{t_{*}, \vartheta}(\cdot), v_{t_{*}, \vartheta}(\cdot)\right) \in W^{1,2}\left(\left[t_{*}, \vartheta\right] ; H\right) \cap L_{2}\left(\left[t_{*}, \vartheta\right] ; V\right)$, $x(t) \in D\left(\varphi_{\alpha}\right) \forall t \in\left[t_{*}, \vartheta\right], t \rightarrow \varphi_{\alpha}(x(t)) \in A C\left(\left[t_{*}, \vartheta\right]\right)$ is called a motion of the system (1) starting from a position $\left(t_{*}, x_{*}\right) \in T \times D\left(\varphi_{\alpha}\right)$ and corresponding to a control $u_{t, \vartheta}(\cdot) \in P_{t, \vartheta}(\cdot)$ and a disturbance $v_{t, \vartheta}(\cdot) \in Q_{t, \vartheta}(\cdot)$. A partition of $T$ is any finite net $\Delta=\left\{\tau_{i}\right\}_{i=0}^{m}$, where $\tau_{0}=t_{0}, \tau_{m}=\vartheta$, $\tau_{i+1}=\tau_{i}+\delta, \delta=\delta(\Delta)$ is a diameter of $\Delta$. Any possible function (multifunction) $\mathcal{U}: T \times H \rightarrow P$ is said to be a feedback strategy. Feedback strategies correct controls at discrete time moments given by some partition of the interval $T$. A solution $x(\cdot)$ of the inequality (1) starting from an initial state $\left(t_{*}, x_{*}\right)$ and corresponding to a piecewise constant control $u^{h}(\cdot)$ (formed by the feedback principle

$$
\left.u^{h}(t)=u_{i} \in \mathcal{U}\left(\tau_{i}, \xi_{i}\right), \quad t \in\left[\tau_{i}, \tau_{i+1}\right), i \in[0: m-1],\left|\xi_{i}-x\left(\tau_{i}\right)\right|_{H} \leq h\right)
$$

and to a disturbance $v_{t_{*}, \vartheta}(\cdot) \in Q_{t_{*}, \vartheta}(\cdot)$ is called an $(h, \Delta)$-motion $x_{\Delta}^{h}\left(\cdot ; t_{*}, x_{*}, \mathcal{U}, v_{t_{*}, \vartheta}(\cdot)\right)$ generated by a positional strategy $\mathcal{U}$ on a partition $\Delta$. Thus, when we write $x_{\Delta}^{h}(\cdot)$, we mean a solution of the inequality (1) constructed by the feedback principle. The set of all $(h, \Delta)$-motions is denoted by $X_{h}\left(t_{*}, x_{*}, \mathcal{U}, \Delta\right)$. It is clear that the set $X_{h}\left(t_{*}, x_{*}, \mathcal{U}, \Delta\right)$ is not empty for $\left(t_{*}, x_{*}\right) \in T \times D\left(\varphi_{\alpha}\right)$.

Thus, the problem under consideration may be formulated in the following way. Let the inequality (1) be considered on the given time interval $T$. Its solution $x(\cdot)=x\left(\cdot ; t_{0}, x_{0}, u_{T}(\cdot), v_{T}(\cdot)\right)$ depends on some control $u_{T} \in P_{T}(\cdot)$ and disturbance $v_{T}(\cdot) \in Q_{T}(\cdot)$. Let us fix a uniform net $\Delta=\left\{\tau_{i}\right\}_{i=0}^{m}, \tau_{0}=t_{0}, \tau_{m}=\vartheta$, with a diameter $\delta=\delta(\Delta)=\tau_{i}-\tau_{i+1}$. Phase states $x\left(\tau_{i}\right)$ are inaccurately measured at the moments $\tau_{i}$. Results of measurements $\xi_{i} \in H$ satisfy the inequalities

$$
\left|\xi_{i}-x\left(\tau_{i}\right)\right|_{H} \leq h, \quad i \in[0: m-1] .
$$

Here $h$ is a value of the level of informational noise. Some prescribed trajectory $x_{*}(t), t \in T$, and a number $\varepsilon>0$ are given. It is required to construct an algorithm of feedback control of inequality (1) providing fulfillment of the following condition. Whatever the unknown disturbance $v_{T}(\cdot) \in Q_{T}(\cdot)$ may be, the deviation of the phase state $x(t)$ from 
the prescribed trajectory $x_{*}(t)$ at all moments $t \in T$ should not exceed the value of $\varepsilon$ provided the values of $h$ and $\delta$ are sufficiently small.

So, the problem (Problem 1) consists in construction of a positional strategy $\mathcal{U}: T \times H \rightarrow P$ with the following properties: whatever the value $\varepsilon>0$ may be, one can indicate (explicitly) numbers $h_{*}>0$ and $\delta_{*}>0$ such that the inequalities

$$
\rho\left(x_{\Delta}^{h}(\cdot), x_{*}(\cdot)\right) \leq \varepsilon \quad \forall x_{\Delta}^{h}(\cdot) \in X_{h}\left(t_{0}, x_{0}, \mathcal{U}, \Delta\right),
$$

are fulfilled uniformly with respect to all measurements $\xi_{i}$ with the properties (2) if $h \leq h_{*}$ and the partition diameter $\delta=\delta(\Delta) \leq \delta_{*}$. Here the symbol $\rho(x(\cdot), y(\cdot))$ denotes the distance from $x(\cdot)$ to $y(\cdot)$ in the uniform metric, i.e.,

$$
\rho(x(\cdot), y(\cdot))=\sup _{t \in T}|x(t)-y(t)|_{H} .
$$

Along with Problem 1, we consider also another problem. Let the following quality criterion of the process be given:

$$
I\left(x_{T}(\cdot), u_{T}(\cdot), v_{T}(\cdot)\right)=\sigma(x(\vartheta))+\int_{t_{0}}^{\vartheta} \chi(t, x(t), u(t), v(t)) d t,
$$

where $\sigma: H \rightarrow R$ and $\chi: T \times H \times U_{p} \times U_{d} \rightarrow R$ are given functions satisfying the local Lipschitz conditions. Introduce the following ordinary differential equation

$$
\dot{p}(t)=\chi(t, x(t), u(t), v(t)) .
$$

A pair $\{x(\cdot), p(\cdot)\}$, where $x(\cdot)$ is a solution of the inequality (1) starting from an initial state $\left(t_{*}, x_{*}\right)$ and $p(\cdot)$ is a solution of the equation (4) starting from an initial state $\left(t_{*}, p_{*}\right)$ corresponding to a piecewise constant control $u^{h}(\cdot)$ (formed by the feedback principle

$$
\begin{gathered}
u^{h}(t)=u_{i} \in \mathcal{U}^{e}\left(\tau_{i}, \xi_{i}, \psi_{i}\right), \quad t \in\left[\tau_{i}, \tau_{i+1}\right), \quad i \in[0: m-1], \\
\left.\left|\psi_{i}-p\left(\tau_{i}\right)\right| \leq h, \quad\left|\xi_{i}-x\left(\tau_{i}\right)\right|_{H} \leq h\right)
\end{gathered}
$$

and to a disturbance $v_{t_{*}, \vartheta}(\cdot) \in Q_{t_{*}, \vartheta}(\cdot)$ is called an $(h, \Delta, \chi)$-motion $z_{\Delta}^{h}(\cdot)=\left\{x_{\Delta}^{h}\left(\cdot ; t_{*}, x_{*}, \mathcal{U}^{e}, v_{t_{*}, \vartheta}(\cdot)\right), p_{\Delta}^{h}\left(\cdot ; t_{*}, p_{*}, \mathcal{U}^{e}, v_{t_{*}, \vartheta}(\cdot)\right)\right\}$ generated by a positional strategy $\mathcal{U}^{e}: T \times H \times R \rightarrow P$ on a partition $\Delta$. The set of all $(h, \Delta, \chi)$-motions is denoted by $Z_{h}^{\chi}\left(t_{*}, x_{*}, p_{*}, \mathcal{U}^{e}, \Delta\right)$. It is clear that the set $Z_{h}^{\varphi}\left(t_{*}, x_{*}, p_{*}, \mathcal{U}^{e}, \Delta\right)$ is not empty for $\left(t_{*}, x_{*}, p_{*}\right) \in T \times D(\varphi) \times R$.

Problem 2 consists in the following. A prescribed value of the criterion, number $I_{*}$, is fixed. It is necessary to construct a positional strategy $\mathcal{U}^{e}: T \times H \times R \rightarrow P$ with the following properties: whatever 
the value $\varepsilon>0$ and disturbance $v_{T}(\cdot) \in Q_{T}(\cdot)$ may be, one can indicate (explicitly) numbers $h_{*}>0$ and $\delta_{*}>0$ such that the inequalities

$$
\left|I\left(x_{\Delta T}^{h}(\cdot), u_{T}^{h}(\cdot), v_{T}(\cdot)\right)-I_{*}\right| \leq \varepsilon
$$

are fulfilled uniformly with respect to all measurements $\xi_{i}$ with the properties (2) and $\psi_{i},\left|\psi_{i}-p_{\Delta}^{h}\left(\tau_{i}\right)\right| \leq h$, if $h \leq h_{*}$ and the partition diameter $\delta=\delta(\Delta) \leq \delta_{*}$. Here $\left\{x_{\Delta}^{h}(\cdot), p_{\Delta}^{h}(\cdot)\right\} \in Z_{h}^{\chi}\left(t_{0}, x_{0}, 0, \mathcal{U}^{e}, \Delta\right)$, $x_{\Delta}^{h}(\cdot)=x\left(\cdot ; t_{0}, x_{0}, \mathcal{U}^{e}(\cdot), v_{T}(\cdot)\right), p_{\Delta}^{h}(\cdot)=p\left(\cdot ; t_{0}, 0, \mathcal{U}^{e}(\cdot), v_{T}(\cdot)\right)$, the control $u^{h}(\cdot)$ is defined by (5).

As one can see from the statement of Problem 2, to solve this problem at moments $\tau_{i}$, it is necessary to know (perhaps, with an error) the realization $p_{\Delta}^{h}\left(\tau_{i}\right)$. To obtain this information, one should know the realization of disturbance $v(t), t \in\left[t_{0}, \tau_{i}\right]$. If the function $\chi$ does not depend on $v$, i.e., $\chi=\chi(t, x, u)$, then the information on the disturbance $v$ is not required. In this case the quality criterion does not depend on $v(\cdot)$ :

$$
I=I\left(x_{T}(\cdot), u_{T}(\cdot)\right)=\sigma(x(\vartheta))+\int_{t_{0}}^{\vartheta} \chi(t, x(t), u(t)) d t
$$

Therefore, in definition of an $(h, \Delta, \chi)$-motion one can assume that $p_{\Delta}^{h}(\cdot)=p_{\Delta}^{h}\left(\cdot ; t_{*}, p_{*}, \mathcal{U}^{e}\right)$ is a solution of the equation

$$
\dot{p}_{\Delta}^{h}(t)=\chi\left(\tau_{i}, \xi_{i}, u_{i}\right), \quad t \in\left[\tau_{i}, \tau_{i+1}\right), \quad t \geq t_{*},
$$

with the initial condition $p_{\Delta}^{h}\left(t_{*}\right)=p_{*}$.

\section{The Algorithm for Solving Problem 1}

Let us indicate the algorithm for solving Problem 1. Introduce sets

$$
\begin{gathered}
f_{u}(t, v)=\bigcup_{u \in P} f(t, u, v), \quad H(t)=\bigcap_{v \in Q} f_{u}(t, v), \\
H(\cdot)=\left\{u(\cdot) \in L_{2}(T ; H): u(t) \in H(t) \quad \text { for a. a. } t \in T\right\} .
\end{gathered}
$$

Let the following condition be fulfilled.

\section{Condition 1.}

a) sets $H(t)$ for all $t \in T$ are nonempty;

b) there exists a control $u_{*}(\cdot) \in H(\cdot)$ such that $x_{*}(\cdot)=x\left(\cdot ; t_{0}, x_{0}, u_{*}(\cdot)\right)$ where the symbol $x\left(\cdot ; t_{0}, x_{0}, u_{*}(\cdot)\right)$ denotes a solution of the variational inequality

$$
\langle\dot{x}(t)+A x(t), x(t)-z\rangle+\varphi(x(t))-\varphi(z) \leq\left(u_{*}(t), x(t)-z\right)
$$


for a. a. $t \in T$ and all $z \in V, \quad x\left(t_{0}\right)=x_{0}$;

c) the saddle point condition is fulfilled:

$\inf _{u \in P} \sup _{v \in Q}(s, f(t, u, v))=\sup _{v \in Q} \inf _{u \in P}(s, f(t, u, v)) \quad$ for any $t \in T, s \in H$.

Let us give two examples of functions $f$ satisfying condition 1c).

1 A function $f$ does not depend on $t$ and is linear with respect to $u$ and $v$, i.e., $f(t, u, v)=B u-C v, B \in \mathcal{L}\left(U_{p} ; H\right), C \in \mathcal{L}\left(U_{d} ; H\right)$. This case was under discussion in [4].

2 Let a control $u$ and a disturbance $v$ be elements of finite-dimensional Euclidean spaces, i.e., $u \in U_{p}=R^{n}, v \in U_{d}=R^{m}$. A mapping $f$ is given according to the following rule: $f(t, u, v)(\eta)=$ $F(t, \eta, u, v)$, where $F(\cdot): T \times \Omega \times R^{n} \times R^{m} \rightarrow R$ possesses the Carathéodory property: a) for all $\eta \in \Omega$ the function $F_{\eta}(t, u, v)=$ $F(t, \eta, u, v)$ satisfies the Lipschitz property; b) for all $(t, u, v) \in T \times$ $P \times Q$ the function $F_{t, u, v}(\eta)=F(t, \eta, u, v)$ is Lebesgue measurable.

Condition 1c) is fulfilled if either a control $u$ and a disturbance $v$ are separated, i.e., $F(t, \eta, u, v)=F(t, \eta, u)+F(t, \eta, v)$, or the function $F$ is of the following structure: $F(t, \eta, u, v)=\sum_{j=1}^{N} \omega_{j}(\eta) F_{j}(t, u, v)$, where $\omega_{j} \in L_{2}(\Omega), j \in[1: N]$, and the vector function

$$
f_{*}(t, u, v)=\left\{F_{1}(t, u, v), F_{2}(t, u, v), \ldots, F_{N}(t, u, v)\right\}
$$

satisfies the saddle point condition in a "small game" [3]:

$$
\min _{u \in P} \max _{v \in Q} s^{\prime} f_{*}(t, u, v)=\max _{v \in Q} \min _{u \in P} s^{\prime} f_{*}(t, u, v) \quad \text { for any } t \in T, \quad s \in R^{N} .
$$

Here the symbol " $"$ " "prime") means transposition.

Let us describe the procedure of forming an (h, $\Delta)$-motion $x_{\Delta}^{h}\left(t ; t_{0}, x_{0}, \mathcal{U}, v_{t_{0}, t}(\cdot)\right)$ corresponding to a fixed partition $\Delta$ and a strategy $\mathcal{U}$ of the form:

$$
\begin{gathered}
\mathcal{U}(t, x)=\left\{u^{e} \in P: \sup _{v \in Q}\left(x-x_{*}(t), f\left(t, u^{e}, v\right)\right) \leq\right. \\
\left.\inf _{u \in P} \sup _{v \in Q}\left(x-x_{*}(t), f(t, u, v)\right)+h\right\} .
\end{gathered}
$$

Before the start of the work of the algorithm, we fix a value $h \in(0,1)$ and a partition $\Delta=\left\{\tau_{i}\right\}_{i=0}^{m}$ with a diameter $\delta=\delta(\Delta)$. Then we organize the process of control of the system (1) according to the feedback principle in such a way that the motion $x_{\Delta}^{h}(\cdot)=x\left(\cdot ; t_{0}, x_{0}, \mathcal{U}, v_{T}(\cdot)\right)$ remains in a 
sufficiently small neighborhood of $x_{*}(\cdot)$ at all moments $t \in T$ for sufficiently small $h$ and $\delta$ under the action of any disturbance $v_{T}(\cdot) \in Q_{T}(\cdot)$, i.e., the inequality (3) is valid. The work of the algorithm is divided into $(m-1)$ identical steps. In the interval $\left[t_{0}, \tau_{1}\right)$ we assume

$$
u^{h}(t)=u_{0} \in \mathcal{U}\left(t_{0}, x_{0}\right)=P, \quad t \in\left[t_{0}, \tau_{1}\right) .
$$

Under the action of this control as well as of an unknown disturbance $v_{t_{0}, \tau_{1}}(\cdot)$ some $(h, \Delta)$-motion $\left\{x_{\Delta}^{h}\left(\cdot ; t_{0}, x_{0}, \mathcal{U}, v_{t_{0}, \tau_{1}}(\cdot)\right)\right\}_{t_{0}, \tau_{1}}$ is realized. At the moment $t=\tau_{1}$ we determine $u_{1}$ from the condition

$$
u_{1} \in \mathcal{U}\left(\tau_{1}, \xi_{1}\right), \quad\left|\xi_{1}-x_{\Delta}^{h}\left(\tau_{1}\right)\right|_{H} \leq h,
$$

i.e., $u^{h}(t)=u_{1}$ for $t \in\left[\tau_{1}, \tau_{2}\right)$. Then we calculate the realization of the $(h, \Delta)$-motion $\left\{x_{\Delta}^{h}\left(\cdot ; \tau_{1}, x_{\Delta}^{h}\left(\tau_{1}\right), \mathcal{U}, v_{\tau_{1}, \tau_{2}}(\cdot)\right)\right\}_{\tau_{1}, \tau_{2}}$. Let the $(h, \Delta)$-motion $x_{\Delta}^{h}(\cdot)$ be defined in the interval $\left[t_{0}, \tau_{i}\right]$. At the moment $t=\tau_{i}$ we assume

$$
u_{i} \in \mathcal{U}\left(\tau_{i}, \xi_{i}\right), \quad\left|\xi_{i}-x_{\Delta}^{h}\left(\tau_{i}\right)\right|_{H} \leq h,
$$

i.e., $u^{h}(t)=u_{i}$ for $t \in\left[\tau_{i}, \tau_{i+1}\right)$. As the result of the action of this control and of an unknown disturbance $v_{\tau_{i}, \tau_{i+1}}(\cdot)$ the $(h, \Delta)$-motion of the system (1) $\left\{x_{\Delta}^{h}\left(\cdot ; \tau_{i}, x_{\Delta}^{h}\left(\tau_{i}\right), \mathcal{U}, v_{\tau_{i}, \tau_{i+1}}(\cdot)\right)\right\}_{\tau_{i}, \tau_{i+1}}$ is realized in the interval $\left[\tau_{i}, \tau_{i+1}\right]$. The indicated above procedure of forming the $(h, \Delta)$-motion stops at the moment $\vartheta$.

Theorem 1 The strategy $\mathcal{U}(t, x)$ of the form (6) solves the Problem 1.

Proof. Let a partition $\Delta=\left\{\tau_{i}\right\}_{i=0}^{m}$ of the interval $T$ with a diameter $\delta(\Delta)=\delta$ and a value of the level of informational noise $h$ be fixed. Let us estimate the evolution of the function

$$
\varepsilon\left(t ; x_{\Delta}^{h}(\cdot), x_{*}(\cdot)\right)=1 / 2\left|x_{\Delta}^{h}(t)-x_{*}(t)\right|_{H}^{2}+\omega \int_{t_{0}}^{t}\left|x_{\Delta}^{h}(\tau)-x_{*}(\tau)\right|_{V}^{2} d \tau
$$

for $t \in T$. Introduce the functional $l(y(\cdot)): W_{*}(T) \rightarrow R$,

$$
l(y(\cdot))=|y(\cdot)|_{C(T ; H)}+|\dot{y}(\cdot)|_{L_{2}(T ; H)}+|y(\cdot)|_{L_{2}(T ; V)} .
$$

Let $\alpha_{*}=\alpha_{0}$, if $\alpha_{0} \geq 0$, and $\alpha_{*}=0$, if $\alpha_{0}<0$. One can prove in a standard way [1] that there exists a number $K_{*}=K_{*}\left(\alpha_{0}, \omega\right)$ such that for any $x_{0} \in D(\varphi), u_{T}(\cdot) \in P_{T}(\cdot), v_{T}(\cdot) \in Q_{T}(\cdot), x(\cdot)=x\left(\cdot ; t_{0}, x_{0}, u_{T}(\cdot), v_{T}(\cdot)\right)$ the inequality

$$
l(x(\cdot)) \leq K_{*}\left(1+\alpha_{*}\left|x_{0}\right|_{H}+\varphi_{\alpha}^{1 / 2}\left(x_{0}\right)+|u(\cdot)|_{L_{2}\left(T ; U_{p}\right)}+|v(\cdot)|_{L_{2}\left(T ; U_{d}\right)}\right)
$$


is true. It is easily seen that for a. a. $t \in\left[\tau_{i}, \tau_{i+1}\right), i \geq 1$, the inequality

$$
\begin{gathered}
\frac{d}{d t} \varepsilon\left(t ; x_{\Delta}^{h}(\cdot), x_{*}(\cdot)\right) \leq \\
\left(f\left(t, u_{i}, v(t)\right)-u_{*}(t), x_{\Delta}^{h}(t)-x_{*}(t)\right)+\alpha_{0}\left|x_{\Delta}^{h}(t)-x_{*}(t)\right|_{H}^{2}
\end{gathered}
$$

holds. Here

$$
u_{i} \in \mathcal{U}\left(\tau_{i}, \xi_{i}\right), \quad\left|\xi_{i}-x_{\Delta}^{h}\left(\tau_{i}\right)\right|_{H} \leq h,
$$

$\left(\xi_{i}\right.$ is an inaccurate measurement of phase state $\left.x_{\Delta}^{h}\left(\tau_{i}\right)\right), v_{\tau_{i}, \tau_{i+1}}(\cdot)$ is an unknown realization of disturbance, the strategy $\mathcal{U}(t, x)$ is determined from (6). It follows from (7)-(9) that

$$
\begin{gathered}
\frac{d}{d t} \varepsilon\left(t, x_{\Delta}^{h}(\cdot), x_{*}(\cdot)\right) \leq\left(f\left(t, u_{i}, v(t)\right)-u_{*}(t), s_{i}\right)_{H}+ \\
\alpha_{0}\left|x_{\Delta}^{h}(t)-x_{*}(t)\right|_{H}^{2}+ \\
k_{1}\left(h+\int_{\tau_{i}}^{t}\left\{\left|\dot{x}_{\Delta}^{h}(\tau)\right|_{H}+\left|\dot{x}_{*}(\tau)\right|_{H}\right\} d \tau\right), \quad t \in \delta_{i}=\left[\tau_{i}, \tau_{i+1}\right), \quad s_{i}=\xi_{i}-x_{*}\left(\tau_{i}\right)
\end{gathered}
$$

Let us define vectors $v_{i}^{e}$ from the conditions

$$
\inf _{u \in P}\left(s_{i}, f\left(\tau_{i}, u, v_{i}^{e}\right)\right) \geq \sup _{v \in Q} \inf _{u \in P}\left(s_{i}, f\left(\tau_{i}, u, v\right)\right)-h .
$$

It is obvious that

$$
u_{*}(t) \in H(t) \subset \bigcup_{u \in P} f\left(t, u, v_{i}^{e}\right), \quad \text { for a. a. } \quad t \in\left[\tau_{i}, \tau_{i+1}\right) .
$$

By virtue of condition 1a) there exists a control $u^{(1)}(t) \in P, t \in \delta_{i}$, such that

$$
f\left(t, u^{(1)}(t), v_{i}^{e}\right)=u_{*}(t) \quad \text { for a. a. } t \in\left[\tau_{i}, \tau_{i+1}\right] .
$$

Using condition 1c) and (11), we deduce that

$$
\begin{gathered}
\left(s_{i}, f\left(\tau_{i}, u_{i}, v(t)\right) \leq \sup _{v \in Q}\left(s_{i}, f\left(t, u_{i}, v\right)\right)+L\left(t-\tau_{i}\right) \leq\right. \\
\inf _{u \in P} \sup _{v \in Q}\left(s_{i}, f(t, u, v)\right)+h+L\left(t-\tau_{i}\right)= \\
\sup _{v \in Q} \inf _{u \in P}\left(s_{i}, f(t, u, v)\right)+h+L\left(t-\tau_{i}\right) \leq \\
\inf _{u \in P}\left(s_{i}, f\left(\tau_{i}, u, v_{i}^{e}\right)\right)+2\left\{h+L\left(t-\tau_{i}\right)\right\} \leq\left(s_{i}, f\left(t, u^{(1)}, v_{i}^{e}\right)\right)+3 h+2 L\left(t-\tau_{i}\right) .
\end{gathered}
$$


Here $L$ is a Lipschitz constant of the function $f(\cdot)$. In this case it follows from (12), (13) that

$$
\left(s_{i}^{*}, f\left(t, u_{i}, v(t)\right)-u_{*}(t)\right) \leq 4 h+2 L\left(t-\tau_{i}\right)
$$

We derive from the inequalities (10), (14)

$$
\begin{gathered}
\varepsilon\left(t ; x_{\Delta}^{h}(\cdot), x_{*}(\cdot)\right) \leq \varepsilon\left(\tau_{i} ; x_{\Delta}^{h}(\cdot), x_{*}(\cdot)\right)+k_{1} \delta\left(h+\delta+\int_{\tau_{i}}^{\tau_{i+1}}\left\{\left|\dot{x}_{\Delta}^{h}(\tau)\right|_{H}+\right.\right. \\
\left.\left.\left|\dot{x}_{*}(\tau)\right|_{H}\right\} d \tau\right)+\alpha_{0}\left|x_{\Delta}^{h}(t)-x_{*}(t)\right|_{H}^{2}, \quad t \in \delta_{i} .
\end{gathered}
$$

Since

$$
\varepsilon\left(t_{0} ; x_{\Delta}^{h}(\cdot), x_{*}(\cdot)\right)=0, \quad \varepsilon\left(\tau_{1} ; x_{\Delta}^{h}(\cdot), x_{*}(\cdot)\right) \leq k_{2}\left(h+\delta^{1 / 2}\right),
$$

by (7), (15), and (16) we have for $t \in T$

$$
\begin{gathered}
\varepsilon\left(t ; x_{\Delta}^{h}(\cdot), x_{*}(\cdot)\right) \leq k_{2}\left(h+\delta^{1 / 2}\right)+k_{1} \delta\left(h\left(\vartheta-t_{0}\right) / \delta+\right. \\
\left.\int_{t_{0}}^{t}\left\{\left|\dot{x}_{\Delta}^{h}(\tau)\right|_{H}+\left|\dot{x}_{*}(\tau)\right|_{H}\right\} d \tau\right)+\alpha_{0} \int_{t_{0}}^{t}\left|x_{\Delta}^{h}(\tau)-x_{*}(\tau)\right|_{H}^{2} d \tau .
\end{gathered}
$$

Here constants $k_{1}$ and $k_{2}$ do not depend on $h, \delta$ and can be explicitly written. The conclusion of the theorem follows from Gronwall's lemma. The theorem is proved.

Remark 1. The trajectory $x_{*}(\cdot)$ plays the role of a "stable path", which is famous in the theory of differential games. By virtue of Theorem 1 , the strategy $\mathcal{U}$ of form (6) guarantees that the solution of inequality (1) follows the trajectory $x_{*}(\cdot)$ (the strategy "leads" $x(\cdot)$ along $x_{*}(\cdot)$ ) irrespective of the unknown effective perturbation. As to the problem of choosing the stable path itself, it is a typical problem of a program control of a parabolic variational inequality and was studied by many authors.

\section{The Algorithm for Solving Problem 2}

Let us indicate the algorithm for solving Problem 2. Denote

$$
\begin{gathered}
\Phi(t, x, u, v)=\{f(t, u, v), \chi(t, x, u, v)\} \\
\Phi_{u}(t, x, v)=\bigcup_{u \in P} \Phi(t, x, u, v), \quad H_{*}(t ; x)=\bigcap_{v \in Q} \Phi_{u}(t, x, v),
\end{gathered}
$$


$H_{*}(\cdot ; x)=\left\{u(\cdot) \in L_{2}(T ; H \times R): u(t) \in H_{*}(t ; x) \quad\right.$ for a. a. $\left.t \in T\right\}$.

Let the following condition be fulfilled.

\section{Condition 2.}

a)there exists a control $u^{*}(\cdot)=\left\{u_{1}(\cdot), u_{2}(\cdot)\right\} \in L_{2}(T ; H \times R), u^{*}(t) \in$ $H_{*}\left(t ; x_{*}(t)\right)$ for a. a. $t \in T$, such that $I_{*}=\sigma\left(x_{*}(\vartheta)\right)+z_{*}(\vartheta)$, where $x_{*}(\cdot)=x\left(\cdot ; t_{0}, x_{0}, u_{1}(\cdot)\right)$, the symbol $x\left(\cdot ; t_{0}, x_{0}, u_{1}(\cdot)\right)$ denotes a solution of the variational inequality

$$
\begin{gathered}
\langle\dot{x}(t)+A x(t), x(t)-z\rangle+\varphi(x(t))-\varphi(z) \leq\left(u_{1}(t), x(t)-z\right) \\
\text { for a. a. } t \in T \text { and all } z \in V, \quad x\left(t_{0}\right)=x_{0},
\end{gathered}
$$

and the symbol $z_{*}(\cdot)$ stands for a solution of the ordinary differential equation

$$
\dot{z}(t)=u_{2}(t), \quad t \in T, \quad z\left(t_{0}\right)=0 ;
$$

b) the saddle point condition is fulfilled:

$$
\inf _{u \in P} \sup _{v \in Q}\{(s, f(t, u, v))+r \chi(t, x, u, v)\}=
$$

$\sup _{v \in Q} \inf _{u \in P}\{(s, f(t, u, v))+r \chi(t, x, u, v)\} \quad$ for any $t \in T, x, s \in H, r \in R$.

Let us describe the procedure of forming an $(h, \Delta, \chi)$-motion $z_{\Delta}^{h}(\cdot)=$ $\left\{x_{\Delta}^{h}(\cdot), p_{\Delta}^{h}(\cdot)\right\}$ corresponding to a fixed partition $\Delta$ and a strategy $\mathcal{U}^{e}$ of the form:

$$
\begin{gathered}
\mathcal{U}^{e}(t, x, p)= \\
\left\{u^{e} \in P: \sup _{v \in Q}\left\{\left(x-x_{*}(t), f\left(t, u^{e}, v\right)\right)+\left(p-z_{*}(t)\right) \chi\left(t, x, u^{e}, v\right)\right\} \leq\right. \\
\left.\inf _{u \in P} \sup _{v \in Q}\left\{\left(x-x_{*}(t), f(t, u, v)\right)+\left(p-z_{*}(t)\right) \chi(t, x, u, v)\right\}+h\right\} .
\end{gathered}
$$

The algorithm for solving Problem 2 is analogous to the algorithm for solving Problem 1. Before the start of algorithm's work, we fix a value $h \in(0,1)$ and a partition $\Delta=\left\{\tau_{i}\right\}_{i=0}^{m}$, with a diameter $\delta=\delta(\Delta)$. The work of the algorithm is subdivided into $(m-1)$ identical steps. In the interval $\left[t_{0}, \tau_{1}\right)$ we assume

$$
u^{h}(t)=u_{0} \in \mathcal{U}^{e}\left(t_{0}, x, p\right)=P .
$$

Under the action of this control as well as of an unknown disturbance $v_{t_{0}, \tau_{1}}(\cdot)$, some $(h, \Delta, \chi)$-motion $\left\{z_{\Delta}^{h}(\cdot)\right\}_{t_{0}, \tau_{1}}=\left\{x_{\Delta}^{h}\left(\cdot ; t_{0}, x_{0}, \mathcal{U}^{e}, v_{t_{0}, \tau_{1}}(\cdot)\right)\right.$, $p_{\Delta}^{h}\left(\cdot, t_{0}, 0, \mathcal{U}^{e}, v_{t_{0}, \tau_{t}}(\cdot)\right\}_{t_{0}, \tau_{1}}$ is realized. At the moment $t=\tau_{1}$ we determine $u_{1}$ from the condition

$$
u_{1} \in \mathcal{U}^{e}\left(\tau_{1}, \xi_{1}, \psi_{1}\right), \quad\left|\xi_{1}-x_{\Delta}^{h}\left(\tau_{1}\right)\right|_{H} \leq h, \quad\left|\psi_{1}-p_{\Delta}^{h}\left(\tau_{1}\right)\right| \leq h,
$$


i.e., $u^{h}(t)=u_{1}$ for $t \in\left[\tau_{1}, \tau_{2}\right)$. Then we calculate the realization of the $(h, \Delta, \varphi)$-motion $\left\{z_{\Delta}^{h}(\cdot)\right\}_{\tau_{1}, \tau_{2}}=\left\{x_{\Delta}^{h}\left(\cdot ; \tau_{1}, x_{\Delta}^{h}\left(\tau_{1}\right), \mathcal{U}, v_{\tau_{1}, \tau_{2}}(\cdot)\right), p_{\Delta}^{h}\left(\cdot ; \tau_{1}\right.\right.$, $\left.\left.p_{\Delta}^{h}\left(\tau_{1}\right), \mathcal{U}, v_{\tau_{1}, \tau_{2}}(\cdot)\right)\right\}_{\tau_{1}, \tau_{2}}$. Let the $(h, \Delta, \chi)$-motion $z_{\Delta}^{h}(\cdot)$ be defined in the interval $\left[t_{0}, \tau_{i}\right]$. At the moment $t=\tau_{i}$ we assume

$$
u_{i} \in \mathcal{U}^{e}\left(\tau_{i}, \xi_{i}, \psi_{i}\right), \quad\left|\xi_{i}-x_{\Delta}^{h}\left(\tau_{i}\right)\right|_{H} \leq h, \quad\left|\psi_{i}-p_{\Delta}^{h}\left(\tau_{i}\right)\right| \leq h,
$$

i.e., $u^{h}(t)=u_{i}$ for $t \in\left[\tau_{i}, \tau_{i+1}\right)$. As the result of the action of this control and of an unknown disturbance $v_{\tau_{i}, \tau_{i+1}}(\cdot)$, the $(h, \Delta, \chi)$-motion of the system (1) $\left\{z_{\Delta}^{h}(\cdot)\right\}_{\tau_{i}, \tau_{i+1}}=\left\{x_{\Delta}^{h}\left(\cdot ; \tau_{i}, x_{\Delta}^{h}\left(\tau_{i}\right), \mathcal{U}, v_{\tau_{i}, \tau_{i+1}}(\cdot)\right), p_{\Delta}^{h}\left(\cdot ; \tau_{i}, p_{\Delta}^{h}\left(\tau_{i}\right)\right.\right.$, $\left.\left.\mathcal{U}, v_{\tau_{i}, \tau_{i+1}}(\cdot)\right)\right\}_{\tau_{i}, \tau_{i+1}}$ is realized in the interval $\left[\tau_{i}, \tau_{i+1}\right]$. The described above procedure of forming the $(h, \Delta, \chi)$-motion stops at the moment $\vartheta$.

THEOREM 2 The strategy $\mathcal{U}^{e}(t, x, p)$ of the form (18) solves the Problem 2.

Scheme of the proof of this theorem is analogous to the proof of Theorem 1. At that we estimate the evolution of the function

$$
\mu\left(t ; x_{\Delta}^{h}(\cdot), p_{\Delta}^{h}(t), x_{*}(\cdot), z_{*}(t)\right)=\varepsilon\left(t, x_{\Delta}^{h}(\cdot), x_{*}(\cdot)\right)+1 / 2\left|p_{\Delta}^{h}(t)-z_{*}(t)\right|^{2} .
$$

Let a partition $\Delta=\left\{\tau_{i}\right\}_{i=0}^{m}$ of the interval $T$ with a diameter $\delta(\Delta)=\delta$ and a value of the level of informational noise $h$ be fixed. Analogous to (10) we deduce for a. a. $t \in\left[\tau_{i}, \tau_{i+1}\right), i \geq 1$, that the inequality

$$
\begin{gathered}
\frac{d}{d t} \mu\left(t ; x_{\Delta}^{h}(\cdot), p_{\Delta}^{h}(t), x_{*}(\cdot), z_{*}(t)\right) \leq \\
\left(f\left(t, u_{i}, v(t)\right)-u_{1}(t), x_{\Delta}^{h}(t)-x_{*}(t)\right)+\alpha_{0}\left|x_{\Delta}^{h}(t)-x_{*}(t)\right|_{H}^{2}+ \\
\left(\chi\left(t, x_{\Delta}^{h}(t), u_{i}, v(t)\right)-u_{2}(t)\right)\left(p_{\Delta}^{h}(t)-z_{*}(t)\right)
\end{gathered}
$$

holds. Here

$$
u_{i} \in \mathcal{U}^{e}\left(\tau_{i}, \xi_{i}, \psi_{i}\right), \quad\left|\xi_{i}-x_{\Delta}^{h}\left(\tau_{i}\right)\right|_{H} \leq h, \quad\left|\psi_{i}-p_{\Delta}^{h}\left(\tau_{i}\right)\right| \leq h,
$$

$v_{\tau_{i}, \tau_{i+1}}(\cdot)$ is an unknown realization of disturbance, the strategy

$$
\mathcal{U}^{e}(t, x, p)
$$

is determined from (18). It follows from (19), (20) and the local Lipschitz property that

$$
\begin{gathered}
\frac{d}{d t} \mu\left(t, x_{\Delta}^{h}(\cdot), p_{\Delta}^{h}(t), x_{*}(\cdot), z_{*}(t)\right) \leq\left(f\left(t, u_{i}, v(t)\right)-u_{1}(t), \xi_{i}-x_{*}\left(\tau_{i}\right)\right)+ \\
\quad\left(\chi\left(\tau_{i}, \xi_{i}, u_{i}, v(t)\right)-u_{2}(t)\right)\left(p_{\Delta}^{h}\left(\tau_{i}\right)-z_{*}\left(\tau_{i}\right)\right)+\alpha_{0}\left|x_{\Delta}^{h}(t)-x_{*}(t)\right|_{H}^{2}+
\end{gathered}
$$




$$
\begin{gathered}
k_{1}\left(h+\omega_{\chi}\left(t-\tau_{i}\right) \int_{\tau_{i}}^{t}\left\{\left|\dot{x}_{\Delta}^{h}(\tau)\right|_{H}+\left|\dot{x}_{*}(\tau)\right|_{H}+\left|\dot{p}_{\Delta}^{h}(\tau)\right|+\left|\dot{z}_{*}(\tau)\right|\right\} d \tau\right), \\
t \in \delta_{i}=\left[\tau_{i}, \tau_{i+1}\right),
\end{gathered}
$$

and constant $k_{1}$ can be explicitly written. Here

$$
\begin{gathered}
\omega_{\chi}(\delta)=\sup \left\{\left|\chi\left(t_{1}, x, u, v\right)-\chi\left(t_{2}, x, u, v\right)\right|: t_{1}, t_{2} \in T,\right. \\
\left.\left|t_{1}-t_{2}\right| \leq \delta, u \in P, v \in Q, x \in X\right\}
\end{gathered}
$$

is the modulo of continuity of a function $\chi(\cdot), X \subset H$ is some bounded domain, which all solutions of the inequality (1) belong to. The conclusion of the theorem follows from Gronwall's lemma. Further argument repeats the one presented in the proof of Theorem 1. At that we take the function $\Phi$ instead of the function $f$. The theorem is proved.

\section{References}

[1] V. Barbu. Optimal control of variational inequalities. Pitman Advanced Publishing Program, London, 1984.

[2] N.N. Krasovskii. Controlling of a dynamical system. Nauka, Moscow, 1985. in Russian.

[3] N.N. Krasovskii and A.I. Subbotin. Game-theoretical control problems. Springer, Berlin, 1988.

[4] V. I. Maksimov. Feedback minimax control for parabolic variational inequality. C.R.Acad.Sci., Paris, Série II b:105-108, 2000.

[5] Ju. S. Osipov. Differential games for systems with hereditary. Dokl. AN USSR, 196(4):779-782, 1971. in Russian. 